\title{
LA COMIDA EN EL ANTIGUO PERÚ: HAKU MIKUMUSUM (IVAMOS A COMER!)
}

\author{
Oswaldo Salaverry ${ }^{1,2, a}$
}

\section{RESUMEN}

La región andina es uno de los ocho centros originarios mundiales de domesticación de plantas y animales. Al ser la alimentación un factor estructurante de la realidad social, se describe la estrecha relación que desarrolló el hombre andino con su medio geográfico, y las plantas y animales que domesticó dando lugar a una cultura singular en la que aunó la extraordinaria riqueza nutricional de sus alimentos tradicionales, con un sistema de producción, conservación y distribución de los alimentos que permitió una civilización en la que no se conoció el hambre. En la siguiente revisión se presenta brevemente algunos alimentos del antiguo Perú.

Palabras clave: Alimentos; Población indígena; Plantas comestibles; Perú (fuente: DeCS BIREME).

\author{
FOOD IN ANCIENT PERU: \\ HAKU MIKUMUSUM (LET'S GO TO EAT!)
}

\begin{abstract}
The Andean region is one of the eight world centers where plant and animal domestication originated. Given that food is a structural factor of social reality, a description is given of the close relationship that Andean men developed with their geographic surroundings, and the plants and animals they domesticated, giving rise to a remarkable culture that united the extraordinary nutritional richness of their traditional food with a food production, conservation and distribution system that led to a civilization who never experienced hunger. Some foods of ancient Peru are briefly described in the following review.
\end{abstract}

Key words: Food; Indigenous population; Plants, edible; Peru (source: MeSH NLM).

\section{ALIMENTACIÓN Y CULTURA}

El desarrollo y la evolución de la cultura humana están estrechamente vinculados con la alimentación. Mas allá de la obviedad de que el alimento es imprescindible para la subsistencia de cualquier especie, los humanos primitivos, al domesticar animales y plantas, artificializaron su relación con el medio ambiente, lograron, a diferencia de cualquier otra especie animal, modular la naturaleza según sus necesidades; es así como inició su desarrollo hacia el dominio hegemónico del planeta. Los ecosistemas en que esto ocurrió se conocen como centros mundiales de domesticación de plantas y animales y fueron, por tanto, la sede de las primeras culturas y civilizaciones desde las cuales se diseminaría por todo el planeta la cultura de la alimentación en sus más variadas formas. Se reconocen ocho centros mundiales originarios de domesticación, denominados Centros Vavílov en honor al científico ruso que los propuso ${ }^{(1)}$. Por razones geográficas la mayoría de estos centros estuvieron en contacto e intercambiaron especies domesticadas desde la prehistoria, a excepción del centro americano, el cual tiene dos subcentros: el andino y el mesoamericano, que tuvieron un desarrollo paralelo y en aislamiento, de las especies domesticadas en Eurasia y África hasta finales del siglo XV.

Con los viajes de Colón se produce un encuentro de dos mundos culturales y alimenticios que, de allí en adelante, confluirían mundializando y homogenizando la alimentación humana, hasta configurar lo que algunos han llamado los "imperios alimenticios" (2). La Organización de las Naciones Unidas para la Alimentación y la Agricultura (FAO) estima que unas 7000 plantas han sido cultivadas o recolectadas como alimento en algún momento de la historia, pero en la actualidad la seguridad

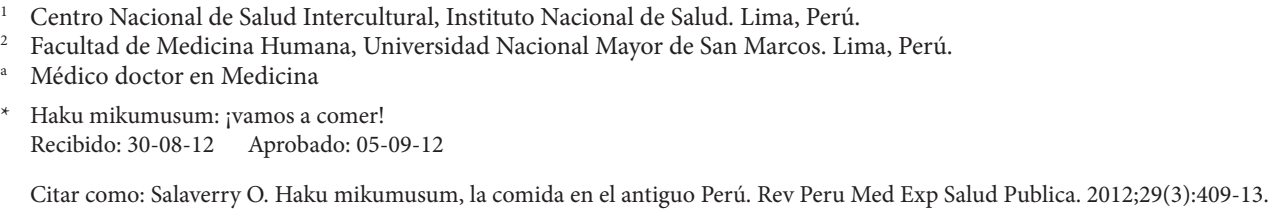


alimentaria reposa en 30 cultivos que aportan el $95 \%$ de las calorías de la dieta, y tres de ellos: el trigo, el arroz y el maíz brindan más del $50 \%$ de las calorías derivadas de las plantas.

La importancia fundamental de los procesos de domesticación de plantas y animales no puede ser soslayada para una cabal comprensión histórica de las culturas originarias, pues de ellos derivan las interpretaciones cosmológicas, los modos de organización del trabajo, y los regímenes de tenencia de los recursos y su distribución. Los modelos culturales que así se crean están tan profundamente enraizados en la vida cotidiana que no suelen ser reconocidos por los integrantes de esas mismas sociedades, pues sus consecuencias y modos de expresión están incorporados en una percepción incuestionable del mundo ${ }^{(3)}$. En los Andes precolombinos la profunda conexión entre animales, plantas y cultura se extendió mucho más allá de la alimentación configurando las tecnologías productivas, la división del tiempo, la cosmogonía, la moral y hasta las formas de la religiosidad. Como lo han desarrollado varios historiadores contemporáneos, la alimentación es un aspecto estructurante de la cultura humana ${ }^{(4,5)}$.

\section{EL PROCESO DE DOMESTICACIÓN ANDINO}

Hace unos 12000 a 10000 años atrás los pobladores andinos eran nómades, recolectores y cazadores de grandes animales, donde destacaba un tipo de oso perezoso y un pariente lejano del actual caballo. La caza intensa extinguió estos animales y se buscó entonces animales más pequeños como los camélidos, venados o ciervos, roedores y aves. Este es el periodo en el cual se inicia también la agricultura eventual, es decir aquella que dejaba semillas de alguna planta silvestre o parcialmente domesticada en un lugar y se continuaba con la trashumancia, regresando luego para recoger la cosecha, pero sin llegar al sedentarismo ${ }^{(6)}$. Entre las primeras plantas domesticadas se encuentra las de la familia Chenopodium, de donde se derivarían las diversas variedades de quinua (Chenopodium quinoa) y de la familia Amaranthaceae de donde derivaría el amaranto o kiwicha (Amaranthus caudatus). Las investigaciones arqueológicas muestran un cambio hace unos 9000 años, cuando se hacen más abundantes los huesos de camélidos, lo que indicaría el naciente predominio de las labores de pastoreo sobre la caza, especialmente de la llama y la alpaca. El patrón sedentario se va imponiendo y se inicia la domesticación de especies animales pequeñas como el cuy (Cavia porcellus) y el pato (Cairina moschata). El proceso de domesticación se acelera de allí en adelante, pero en los andes al igual que en Centroamérica predomina la domesticación de plantas siendo escasa o parcial la de animales. La razón de esta disparidad no se conoce pero sin duda no se debe a la ausencia de animales salvajes que pudieran haber sido domesticados.

Asombra la diversidad de plantas que se domestican en los andes y cómo se incorporan en un sistema agrícola simple pero efectivo ${ }^{(7)}$ que, aunado a un sistema de distribución asombroso para las condiciones de la época, lograron una diversidad alimenticia mucho mayor que en otros centros de domesticación ${ }^{(8)}$. Las tierras cultivadas en el periodo prehispánico sufrieron una disminución considerable con la conquista europea al punto que se calcula que hacia el año 1960, pese a todas las obras de la moderna agricultura, solo se había alcanzado un $70 \%$ de la superficie cultivada en el antiguo Perú (9).

\section{LOS GRANOS ANDINOS}

\section{MAíz}

El maíz (Zea mays) es, sin duda, el cultivo central de la agricultura andina, pero persiste la disputa sobre dónde se domesticó, pues algunos creen que su origen está en la meseta mexicana y de allí se difunde a toda América, pero otros proponen una domesticación dual en los Andes y en México. Sobre la hipótesis de origen mexicano se ha llegado, incluso, al extremo de identificar a la comunidad paxil, en el estado de Veracruz, como cuna de su domesticación. Paxil significa "tierra fértil" $y$, tomando como referencia el "Popol vuh", sería el lugar de origen del maíz ${ }^{(10)}$. Esta hipótesis se debe contrastar con las leyendas que pueden encontrarse en todos los pueblos primitivos, desde América del Norte hasta el extremo sur del continente, en los que se indica con bastante precisión como se introduce su cultivo.

En el caso del antiguo Perú, la crónica del Padre Calancha refiere un mito que remite su origen a Pachacamac. En los primeros tiempos, una pareja primordial no conseguía alimentos suficientes para subsistir, el hombre murió de hambre y la mujer recogía raíces para sobrevivir; en su desesperación levantó los ojos hacia el sol y pidió ayuda. El sol escuchó sus suplicas y con sus rayos la fecundó, y a los cuatro días dio a luz. Pachacamac molesto por recibir menos pleitesía, despedazó al niño y para que la mujer no se quejase por falta de alimento, sembró los dientes del niño del cual brotó el maíz ${ }^{(11)}$.

Además de los mitos, la hipótesis de un origen doble en Mesoamérica y los Andes, se ve favorecida por la gran cantidad de especies que se pueden identificar 
en Perú (más de 35). El nombre originario en quechua es sara, pero existieron otros nombres según características especificas; así, al maíz duro se le llamaba muruchu y el maíz de dos colores misa sara. Estas denominaciones quechuas se han perdido, pero otros vocablos se han incorporado al castellano en versión de peruanismos como "choclo", del quechua ChokIlu que se refería a la mazorca de maíz no maduro; el maíz cocido o "cancha" deriva de camcha o sara chanka. El quechua huminta se mantiene en nuestra culinaria popular como "humita". Otros se mantienen en usos locales como los derivados de tanta que refiriéndose específicamente al pan de maíz se convirtió luego en genérico de pan. Una especial mención debe hacerse al sanku que era una masa de maíz semicocido que se utilizaba en la fiesta de la salud situa o citua y que luego mezclada con sangre de los sacrificios de animales se convertía en el yawar sancu utilizado en forma ritual para proteger la salud ${ }^{(12)}$. La ritualidad alrededor del propio cultivo es abundante, en el periodo de siembra se usaban las saramamas y en la época de cosecha el baile correspondiente a la cosecha de maíz era el ayrigua ${ }^{(12) .}$

La primera noticia que tuvieron los europeos de esta gramínea fue a través de Colón, que observó las plantaciones en las islas del Caribe. Una referencia clara al maíz está en las "Decadas" de Pedro Mártir de Angleria, escrita desde 1493 pero publicada en 1511, aunque para dicha fecha el maíz ya se conocía en toda Europa, apareciendo en el "De historia stirpium" o herbario de Fuchs en $15422^{(13)}$.

\section{LA QUINUA}

La quinua y la cañihua, además de ser especies de Amaranthus, guardan una estrecha relación en su consumo. El origen de la quinua es sin duda altoandino aunque también se han encontrado cultivos en Mesoamérica, en particular el Chenopodium nuttaliae, conocido como huauhtli muy similar a la quina andina. Hallazgos en tumbas y otros restos arqueológicos sitúan la domesticación de la quinua en fechas tan tempranas como el 3000 a. C. aunque se refieren hasta 7000 años de antigüedad ${ }^{(14)}$. Originalmente el grano de la quinua tenía un color oscuro, casi negro (sus granos se llamaban ayaras o ajaras) y por un proceso de selección fue obteniéndose las cosechas de su actual color perlado. Aún se conservan especies de muy diversos colores, lo cual también se refleja en la amplia sinonimia y denominaciones que guarda relación con su amplia distribución. Además, las cenizas de la quinua llipta, forman parte del ritual del chacchado o uso de la hoja de coca para mascar, como mordiente para extraer en la boca las sustancias de la hoja de coca.

\section{LOS TUBÉRCULOS ANDINOS}

\section{LA PAPA}

Conocida también como patata, a diferencia del maíz que tenía un cultivo centralizado, era de uso popular, sirviendo incluso para medir el tiempo calculando el equivalente al que tomaba hervir una olla de papas, o para medir una superficie a través de la papacancha o topo que era la extensión de terreno que debía tener una familia para sembrar papa, lo que variaba según la altitud y el consiguiente rendimiento del tubérculo; así, en tierras altas que debían descansar más tiempo entre cosechas, un topo era siete a diez veces más grande que el ubicado en una altura media. El rol central de la papa se grafica en la calificación general de papa como kawsay es decir subsistencia o vida.

Un viejo relato andino refleja las tensiones entre cultivadores de quinua y de papa; se refiere que en la antigüedad los cultivadores de quinua dominaban todo el territorio y condenaban a morir a sus vasallos reduciéndoles los alimentos, estos clamaron a la divinidad que les dio una semillas semejantes a piedras las que comenzaron a cultivar, los opresores les dejaron cultivar pero cuando la cosecha estaba lista llegaron y se llevaron todo lo que estaba sobre la superficie: las matas de flores moradas de la papa, creyendo así condenarlos a muerte pero el dios les dijo que escarbaran en el suelo y encontraron las papas que mantuvieron en secreto y continuaron cultivando hasta que pudieron vencer a sus opresores.

La conservación de la papa, bajo la forma de chuño dio una ventaja extraordinaria para la conservación y distribución de alimentos, el proceso es una de las tecnologías andinas más sofisticadas y ampliamente difundidas, se iniciaba con la selección de las papas, las que se dejan a la intemperie para que se congelen, luego se las deja en una laguna o cocha durante unos 30 días hasta que, saturadas de agua, se retiran, se extienden sobre el suelo y se las pisa para extraer el agua y la cáscara, luego se las vuelve a exponer al frío por 10 a 15 días, terminando de secarse al sol por la acción del hielo. El producto o chuño, se conserva durante meses o años sin corromperse, permitiendo su distribución o almacenamiento. Este proceso implica un conocimiento profundo de los ecosistemas para su aprovechamiento; así, las papas de las alturas que necesitan protegerse de los rigores del frío, lo logran a costa de brindar una papa pequeña y amarga por su contenido de glicoalcaloides que le dan resistencia a las bajas temperaturas. Estos glicoalcaloides son solubles en agua pero estables frente al calor por tanto no se eliminan con la cocción lo que hace a las papas desagradables, en tanto que procesadas como chuño son altamente valoradas. 


\section{LA OCA}

Cuyo nombre científico es Oxalis tuberosa, se conoce también con diversos nombres en el área andina: cuiba o quiba en Venezuela; macachín o miquichi en Argentina, y huasisai o "papa roja" en Colombia (15). Se usa cocida pero previamente es asoleada para incrementar sus propiedades, en particular su dulzura. Su alto contenido de agua, casi $80 \%$, ha llevado a que se la use deshidratada por exposición al sol, tomando el nombre de ccaya lo que lleva su porcentaje proteico hasta el $11 \%$. Una expresión de su vinculación con otros cultivos es el tradicional sistema de rotación, en el cual un mismo campo tiene una cosecha de papa y a continuación se realiza una de oca.

\section{LA ARRACACHA}

De amplia distribución en los Andes, desde Venezuela hasta Bolivia, ha dado lugar a una variada sinonimia; así, en Ecuador se la conoce como zanahoria blanca, en Estados Unidos y Europa como white carrot; en Venezuela se le llama apio criollo; en el periodo prehispánico, en quechua se la conocía como rakkacha o también huisampilla, en aymara se la denominaba lakachu o lecachu. En el Perú se identifican dos centros de diversidad genética, uno en la sierra norte y otro en la sierra sur; la planta es una herbácea que alcanza hasta $1 \mathrm{~m}$ con hojas amplias lanceoladas, flores púrpuras o grises, su raíz alcanza hasta $4 \mathrm{~kg}$ de peso y es la parte comestible, el color varía desde amarilla hasta morada y se ramifica en 8 a 10 partes ${ }^{(16)}$.

\section{LEGUMINOSAS}

La principal leguminosa domesticada en los Andes fue el frejol de la cual se desarrollaron múltiples especies y variedades. La domesticación se realizó a partir de una variedad salvaje que existe en toda América. Algunas subespecies como la ñuña (Phaseolus vulgaris subesp nunas) solo crecen en los Andes. Las otras leguminosas como el tarwi (Lupinus mutabilis) han sido muy afectadas por el desplazamiento de cultivos con plantas introducidas, y habiendo sido uno de los principales cultivos precolombinos ${ }^{(17)}$ su consumo actualmente es casi doméstico en regiones andinas aunque sus valores nutricionales son notables con un $42 \%$ de proteína en grano seco y $20 \%$ en grano cocido.

El frejol es una planta del género Phaseolus con diversas especies distribuidas en toda América, la especie más común, el Phaseolus vulgaris L., es originario de los Andes aunque aún se debate su posible origen mesoamericano. Las evidencias remontan su antigüedad hacia el
7000 a.C. En quechua se le llamaba purutu, de donde deriva el término poroto. Los europeos las llamaron favones o favas por la semejanza con las habas, pero la sinonimia en España es muy amplia: habichuela (que deriva de haba), alubia, que deriva del árabe al-lubiia, o judías del árabe yudiya, vinculado con el iudaeus o judío en latín y que guarda relación con el hecho que los cultivos en la España medieval estaban muy vinculados con la población árabe, pero la distribución a los comerciantes judíos.

\section{ANIMALES DOMÉSTICOS}

No sería completa una revisión de la alimentación precolombina sin mencionar los animales domésticos, entre ellos destaca el cuy, nombre con el que se le conoce en los andes sudamericanos, siendo general la denominación de conejillo de indias o cobayo. En quechua se le llamaba quwe o akash, en aimara wanko y en jakaru kiucho o uywa. Los españoles que no conocían otro roedor semejante le llamaron directamente rata o en algunos casos cochinillo de indias.

El cuy actual (Cavia porcellus cobaya) es originario de Perú, y aunque todos lo suponen un roedor, existe una discusión al respecto ${ }^{(18)}$. El derivado "conejillo de indias", ha trascendido pero también ha tenido inexplicables derivaciones como en ingles en que se le conoce como guinea pig, es decir cerdo de guinea. Aunque su crianza estaba difundida en todo el antiguo Perú incluyendo la costa y la zona altiplánica, la mayor concentración fue en la zona andina, en la que se conserva desde tiempos prehispánicos, un carácter de crianza doméstica.

Los camélidos andinos se utilizaban básicamente por su lana pero también por su carne; los grandes rebaños se atrapaban en chacos, que eran grandes actividades colectivas que consistían en trazar un cerco humano alrededor de una zona e ir reduciéndolo mientras se hacía ruidos que iban concentrando a los rebaños; atrapados en un pequeño espacio se esquilaba a los animales y se sacrificaba una cantidad estrictamente regulada para obtener carne que luego se preservaba por un proceso basado en la sal, la exposición a las bajas temperaturas y el ambiente seco de las alturas andinas. El producto que se obtenía, el charqui, conservaba un gran valor nutricional pues tiene proteínas de alta calidad y muy pocas grasas además de poder conservarse y trasladarse con gran facilidad.

A la llegada de los españoles se continuó con los chacos, pero se perdió paulatinamente ese control que regulaba la sostenibilidad de los rebaños, lo cual 
aunado a la epidemia que afectó a todos los camélidos andinos llevó a una gran disminución que afectó el sistema de transporte de mercancías, pero básicamente de alimentos que había funcionado perfectamente durante siglos, incluso antes de los incas. Como dato curioso cabe señalar que los españoles nunca fueron muy afectos a la carne de los camélidos andinos pero apreciaban mucho unas concreciones calcáreas muy frecuentes en el sistema digestivo de estos animales que asimilaron a la piedra bezoar, un componente de múltiples antídotos de la farmacopea medieval y que alcanzaba altos precios en Europa donde se obtenía de camélidos asiáticos ${ }^{(12)}$.

\section{LA ALIMENTACIÓN DEL POBLADOR ANDINO}

Aunque solo hemos señalado algunas de las plantas alimenticias del antiguo Perú, la organización de la producción, el sistema de distribución centralizado y la sostenibilidad de la agricultura andina permitió que, según referencias de los cronistas de indias, no se conociera el hambre en el periodo precolombino, y por el contrario se disfrutara de una amplia diversidad de alimentos, el valor de los cultivos andinos tradicionales recién se está valorando no solo por su diversidad y aporte nutricional sino por su contribución a la configuración de una nación andina y agrícola como nuestra principal característica.

\section{REFERENCIAS BIBLIOGRÁFICAS}

1. Vavilov NI. Origin and Geography of cultivated plants. Cambridge: Cambridge University Press; 1992.

2. Fraser EDG, Rimas A. Empires of food. New York: Free Press; 2010.

3. Levy-Strauss C, Mitológicas I. Lo crudo y lo cocido. México: Fondo de Cultura Económica; 2005.

4. McNeill W. Plagues and people. New York: Anchor Books1989.

5. McNeill W. The Human condition: An ecological and historical view. Princenton: Princenton University Press; 1980.

6. Harris M, Ross E (ed.) Food and evolution: Toward a theory of human food habits. Philadelphia: Temple University Press; 1987.

7. Guaman Poma de Ayala F. Nueva crónica y buen gobierno (Codex péruvien illustré). Paris: Institut d'ethnologie Université de Paris; 1936.

8. Pearsall D. The origins of plant cultivation in South America. En: Cowan C, Watson P. The origins of agriculture. An International perspective. Alabama: University of Alabama Press: 2006. p. 173-206.
9. Kosok P. Life, land and water in ancient Peru: an account of the discovery, exploration and mapping of ancient pyramids, canals, roads, towns, and fortresses of coastal Peru with observations of various aspects of Peruvian life, both ancient and modern. New York: Long Island University Press; 1965.

10. Navarrete Cáceres C. Relatos mayas de tierras altas sobre el origen del maíz: el camino de Paxil. México: UNAM; 2002.

11. Rostworowski M. Obras completas II Pachacamac. Lima: Instituto de estudios Peruanos; 2002.

12. Lastres J. Historia de la Medicina Peruana. Lima: Universidad Nacional Mayor de San Marcos; 1955.

13. Frances Causape M. El renacimiento una nueva era para la Materia Medica. En: Jarava J. Historia de las yerbas y plantas (editado por Maria Jesus Mancho) Salamanca: Universidad de Salamanca; 2005. p. 29.

14. Pearsall DM. The origins of plant cultivation in South America. In: Wesley Cowan C, Watson PJ (eds.). The origins of agriculture: An international perpective. Washington; Smithsonian Institution Press; 1992. p. 173-205.

15. Marrou L, Villacorta M, Pagador S. Composición química de "oca" (Oxalis tuberosa), "arracacha” (Arracaccia xantorhorriza) y "tarwi" (Lupinus tuberosa). Formulación de una mezcla base para productos alimenticios. Rev Venez Cienc Tecnol. 2011;2(2):239-52.

16. Amaya J. Arracacha. La Libertad: Gobierno Regional La Libertad. 2006.

17. Horkheimer H. Alimentación y obtención de alimentos en el Perú Prehispánico. Lima: Universidad Nacional Mayor de San Marcos; 1973. p. 97-100.

18. Graur D, Hide WA, Li WH. Is the guinea-pig a rodent? Nature. 1991;351(6328):649-52.

\footnotetext{
Correspondencia: Oswaldo Salaverry

García

Dirección: Cápac Yupanqui 1400, Lima

11, Perú.

Teléfono: (511) 6176200 anexo 1609

Correo electrónico: oswaldosalaverry@gmail. com
} 